\title{
Analytic Model Predictive Controller in Simple Symbolic Form
}

\author{
Daniel Honc and Milan Jičínský \\ University of Pardubice, nám. Čs. legií 565, 53002 Pardubice, Czech Republic \\ daniel.honclupce.cz
}

\begin{abstract}
Paper deals with an analytic solution of Model Predictive Controller in simple symbolic form. Process is approximated with a first order dynamical model. Special choice of prediction and control horizons is considered, so the symbolic solution is still applicable, and the controller has interesting "predictive" feature in case of known future set-point course. Such a controller can be used in simple devices like PLCs or microcontrollers without need of matrix operations. Its advantage is that the controller reacts to the process model parameters and penalty parameter change so the control can be very fast and efficient even in adaptive manner.
\end{abstract}

Keywords: Model Predictive Controller, MPC, first order process model, analytical solution, symbolic.

\section{Introduction}

Model Predictive Control (MPC) is very spread and popular time-domain optimizationbased controller design methodology. Plenty different process models, cost functions (performance indexes), analytical and numerical solutions with lot of choices and parameters give arise huge family of methods studied from theoretical point of view but also being applied in industry in different versions for decades. Strong potential of MPC methods lies in natural and graspable formulation (MPC origins can be found in industry), ability to control large systems with constraints and transport delays and work with known future disturbances or set-points - see [1] and [2].

Connections between MPC and existing analytic control methods has been published in [3]. Standard analytic control methods can be considered as a special case of MPC. Both methods are identical in unconstrained case but MPC does not exhibit poor performance of analytical control methods when a constraint is present. In [4] class of nonlinear and linear plants for which MPC admits an analytical solution was characterized. Optimal control sequence takes significantly less time to calculate in case of analytical solution. On the other hand, quadratic programming can handle different types of constraints.

Presented approach is slightly different - we want to get analytical MPC which is parametrized with model parameters and penalty parameter and does not require any matrix operations or numerical methods. Then such a controller can be used in simple 
systems with less memory and computational power. Because of its parametric feature the controller reacts to process changes (changes in model parameters) and penalty parameter so the user can tune the controller online iteratively or use it in adaptive manner. The complexity of analytical controller depends on choice of prediction and control horizons. The challenge was to find such horizons that the formula is still simple and the controller has predictive behavior - it will react to the set-point change in advance.

\section{Controller derivation}

\subsection{Performance index}

Control aim is set-point following and disturbance rejection together with performance index minimization - to minimize sum of the squares of the future control error and future control changes (control moves)

$$
J=\sum_{j=N_{1}}^{N_{2}}(\hat{y}(k+j)-w(k+j))^{2}+q \sum_{j=1}^{N_{u}} \Delta u(k+j-1)^{2} .
$$

Controlled variable predictions based on actual state and future control actions are needed. We will use dynamical process model for derivation of such a predictor.

\subsection{Process model and predictor}

We consider first order process model with time constant $T$ and gain $Z$

$$
T \frac{d y}{d t}+y=Z u
$$

After discretization with sample time $T_{\mathrm{s}}$ we get discrete-time process model

$$
y(k)+a_{1} y(k-1)=b_{1} u(k-1), \quad a_{1}=-e^{\frac{-T_{s}}{T}}, \quad b_{1}=Z\left(a_{1}+1\right) .
$$

We suppose disturbance model as a random walk process - summation of correlated prediction error $e$ by polynomials $C / A$. The result is that the controller has integrating character.

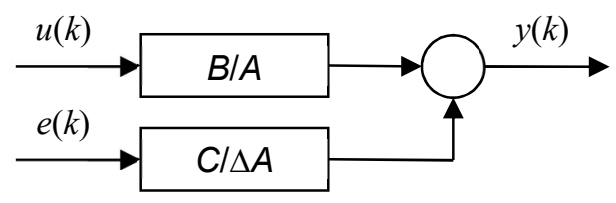

Fig. 1. Discrete-time process and disturbance model 
The process model equation is

$$
y(k)=\frac{B}{A} u(k)+\frac{C}{\Delta A} e(k),
$$

where prediction error $e(k)=y(k)-\hat{y}(k)$ and process polynomials $A=1+a_{1} z^{-1}$ and $B=b_{1} z^{-1}$, second order filtering polynomial $C=1+c_{1} z^{-1}+c_{2} z^{-2}$ and $\Delta A=\left(1+a_{1} z^{-1}\right)\left(1-z^{-1}\right)=1+\left(a_{1}-1\right) z^{-1}-a_{1} z^{-2}$. The $\Delta$ is backward difference operator $\Delta=1-z^{-1}$. By multiplying (4) with $\Delta A$ we get prediction equations in matrix form as

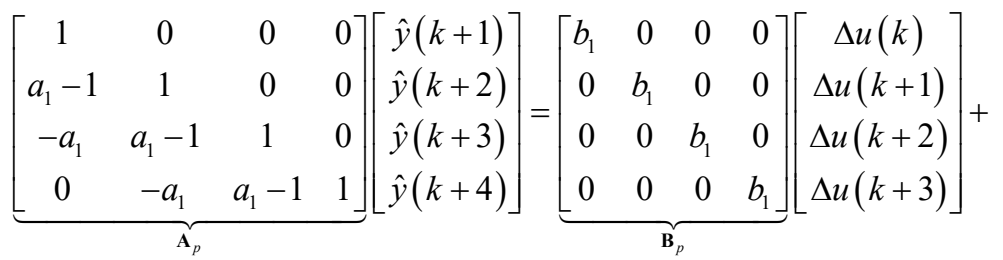

$$
\begin{aligned}
& +\underbrace{\left[\begin{array}{cc}
-a_{1}+1 & a_{1} \\
a_{1} & 0 \\
0 & 0 \\
0 & 0
\end{array}\right]}_{\mathbf{A}_{m}}\left[\begin{array}{c}
y(k) \\
y(k-1)
\end{array}\right]+\underbrace{\left[\begin{array}{cc}
c_{1} & c_{2} \\
c_{2} & 0 \\
0 & 0 \\
0 & 0
\end{array}\right]}_{\mathbf{C}_{m}}\left[\begin{array}{c}
e(k) \\
e(k-1)
\end{array}\right]
\end{aligned}
$$

We suppose the prediction and control horizons $N_{1}=1, N_{2}=N_{\mathrm{u}}=4$ and optimal predictions - zero future prediction error. By multiplying (5) with $\mathbf{A}_{\mathrm{p}}{ }^{-1}$ we get predictor in matrix form as

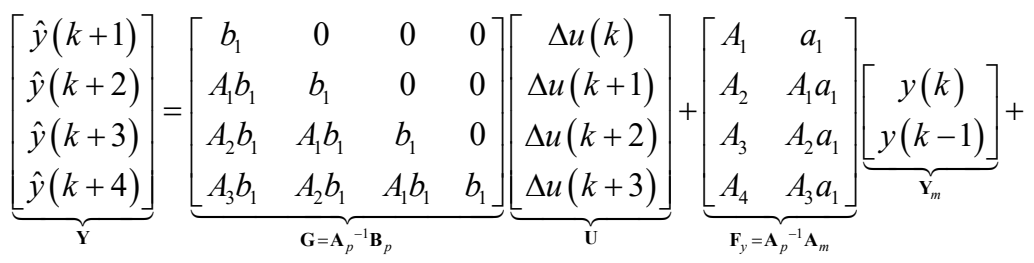

$$
\begin{aligned}
& +\underbrace{\left[\begin{array}{cc}
c_{1} & c_{2} \\
A_{1} c_{1}+c_{2} & A_{1} c_{2} \\
A_{2} c_{1}+A_{1} c_{2} & A_{2} c_{2} \\
A_{3} c_{1}+A_{2} c_{2} & A_{3} c_{2}
\end{array}\right]}_{\mathbf{F}_{e}=\mathbf{A}_{p}^{-1} \mathbf{C}_{m}} \underbrace{\left[\begin{array}{c}
e(k) \\
e(k-1)
\end{array}\right]}_{\mathbf{E}_{m}} \\
& A_{1}=-a_{1}+1, \quad A_{2}=a_{1}^{2}-a_{1}+1, \quad A_{3}=-a_{1}^{3}+a_{1}^{2}-a_{1}+1, \quad A_{4}=a_{1}^{4}-a_{1}^{3}+a_{1}^{2}-a_{1}+1
\end{aligned}
$$




$$
\mathbf{Y}=\mathbf{G} \mathbf{U}+\mathbf{F}_{y} \mathbf{Y}_{m}+\mathbf{F}_{e} \mathbf{E}_{m}=\underbrace{\mathbf{G U}}_{\text {forced response }}+\underbrace{\left[\begin{array}{ll}
\mathbf{F}_{y} & \mathbf{F}_{e}
\end{array}\right]}_{\text {free response } \mathbf{f}} \cdot \underbrace{\left[\begin{array}{c}
\mathbf{Y}_{m} \\
\mathbf{E}_{m}
\end{array}\right]}_{\mathbf{x}_{p}} .
$$

\subsection{Performance index in matrix form and analytic solution}

Cost function (1) in matrix form can be written as

$$
J=(\mathbf{Y}-\mathbf{W})^{T}(\mathbf{Y}-\mathbf{W})+\mathbf{U}^{T} \mathbf{Q} \mathbf{U},
$$

where

$$
\mathbf{W}=\left[\begin{array}{c}
w(k+1) \\
w(k+2) \\
w(k+3) \\
w(k+4)
\end{array}\right], \quad \mathbf{Q}=\left[\begin{array}{cccc}
q & 0 & 0 & 0 \\
0 & q & 0 & 0 \\
0 & 0 & q & 0 \\
0 & 0 & 0 & q
\end{array}\right] .
$$

By substitution (7) into (8) we get following quadratic form

$$
J=\mathbf{U}^{T} \underbrace{\left(\mathbf{G}^{T} \mathbf{G}+\mathbf{Q}\right)}_{\mathbf{H}} \mathbf{U}+\mathbf{U}^{T} \underbrace{\mathbf{G}^{T}(\mathbf{f}-\mathbf{W})}_{\mathbf{g}}+\underbrace{(\mathbf{f}-\mathbf{W})^{T} \mathbf{G}}_{\mathbf{g}^{T}} \mathbf{U}+\underbrace{(\mathbf{f}-\mathbf{W})^{T}(\mathbf{f}-\mathbf{W})}_{k}
$$

and the unconstrained solution can be written as

$$
\mathbf{U}=-\mathbf{H}^{-1} \mathbf{g}=\left(\mathbf{G}^{T} \mathbf{G}+\mathbf{Q}\right)^{-1} \mathbf{G}^{T}\left(\mathbf{W}-\mathbf{F}_{p} \mathbf{x}_{p}(k)\right)=\mathbf{L}\left(\mathbf{W}-\mathbf{F}_{p} \mathbf{x}_{p}(k)\right) .
$$

The control law (actual control change) is

$$
\Delta u(k)=\mathbf{K}\left(\mathbf{W}-\mathbf{F}_{p} \mathbf{x}_{p}(k)\right),
$$

where $\mathbf{K}$ is the first row of matrix $\mathbf{L}$. This is analytic form of predictive controller but matrix operations must be used to calculate $\mathbf{K}$ and $\mathbf{F}_{p}$.

\subsection{Simple symbolic forms of predictive controller}

Simple MPC symbolic forms can be obtained for special choices of prediction and control horizons. If $N_{1}=N_{2}=3$ and $N_{\mathrm{u}}=1$ we get control law as

$$
\begin{aligned}
& \Delta u(k)=\frac{p b_{1}}{q+p^{2} b_{1}^{2}} {\left[w(k+3)+\left(a_{1}^{3}-a_{1}^{2}+a_{1}-1\right) y(k)-a_{1} p y(k-1)+\right.} \\
&\left.+\left(-c_{1} p+c_{2}\left(a_{1}-1\right)\right) e(k)-c_{2} p e(k-1)\right]
\end{aligned}
$$




$$
p=a_{1}^{2}-a_{1}+1
$$

There is only one point in time $k+3$ considered for the set-point following. Only one control change is considered - control action is supposed to be constant for whole control horizon.

For horizons $N_{1}=N_{2}=4$ and $N_{\mathrm{u}}=1$ we get

$$
\begin{gathered}
\Delta u(k)=\frac{r b_{1}}{q+r^{2} b_{1}^{2}}\left[-w(k+4)+\left(a_{1}^{4}-a_{1}^{3}+a_{1}^{2}-a_{1}+1\right) y(k)-\right. \\
\left.-a_{1} r y(k-1)-\left(c_{1} r-c_{2}\left(a_{1}^{2}-a_{1}+1\right)\right) e(k)-c_{2} r e(k-1)\right] \\
r=a_{1}^{3}-a_{1}^{2}+a_{1}-1
\end{gathered}
$$

Controlled variable prediction used to calculate prediction error $e(k)=y(k)-\hat{y}(k)$ is the same for both versions

$$
\hat{y}(k)=b_{1} \Delta u(k-1)-\left(a_{1}-1\right) y(k-1)+a_{1} y(k-2)+c_{1} e(k-1)+c_{2} e(k-2) .
$$

\section{Control experiments}

Firstly we simulated control with model of the laboratory system GUNT RT 050 - speed control (see Fig. 2) - motor with mass flywheel and generator.

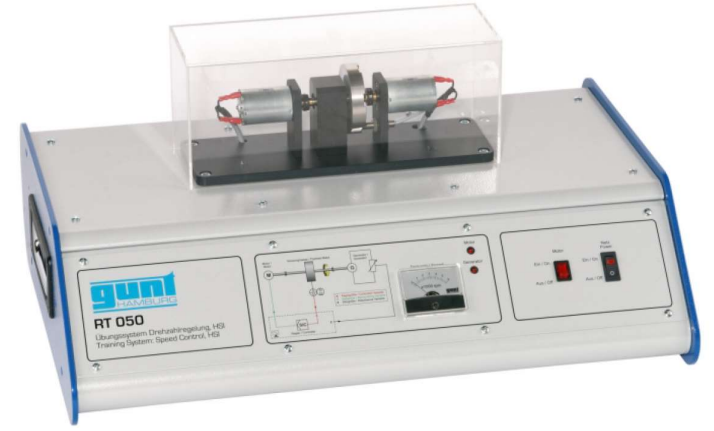

Fig. 2. GUNT RT 050 - speed control laboratory system

We have identified first order continuous-time transfer function model from measured dynamical responses as

$$
F_{1}=\frac{1.96}{3.16 s+1}
$$


With sample time $T_{\mathrm{s}}=0.5 \mathrm{~s}$ and penalty parameter $q=1$ we got following control responses for both versions of the controller - by using equations $(12-16)$.
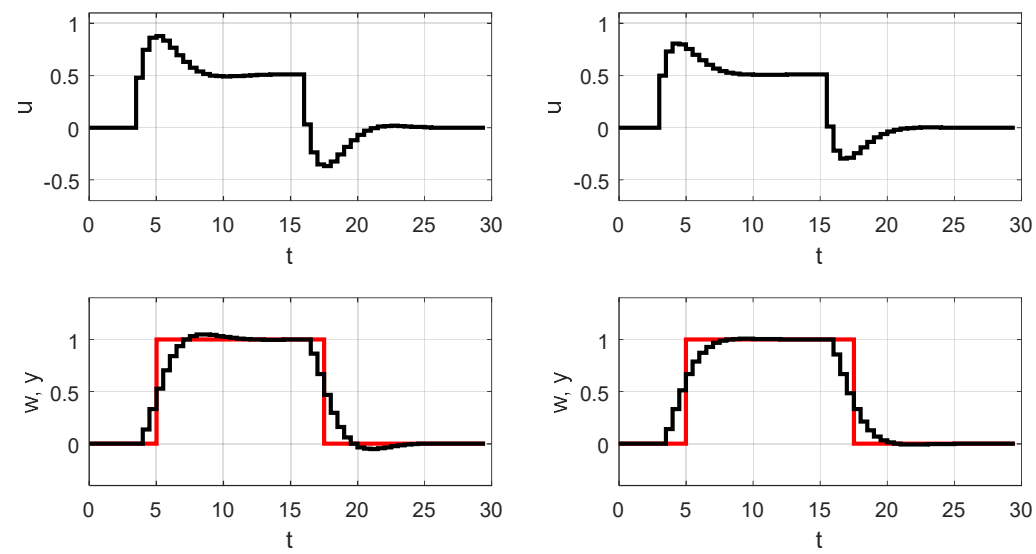

Fig. 3. Simulated control responses with horizons 3 (left) and 4 (right)

We have also tested how the model mismatch and measurement noise will influence the control responses. We have identified second order model of the same system - this is the right order of the controlled process

$$
F_{2}=\frac{1.95}{(2.36 s+1)(0.71 s+1)}
$$

Second version of the controller $\left(N_{1}=N_{2}=4\right)$ uses model $F_{1}(17)$ like first order approximation of the real process. For simulation purpose we have added normally distributed pseudorandom noise with standard deviation 0.01 to the controlled output of the process $F_{2}(18)$ to emulate measurement noise.
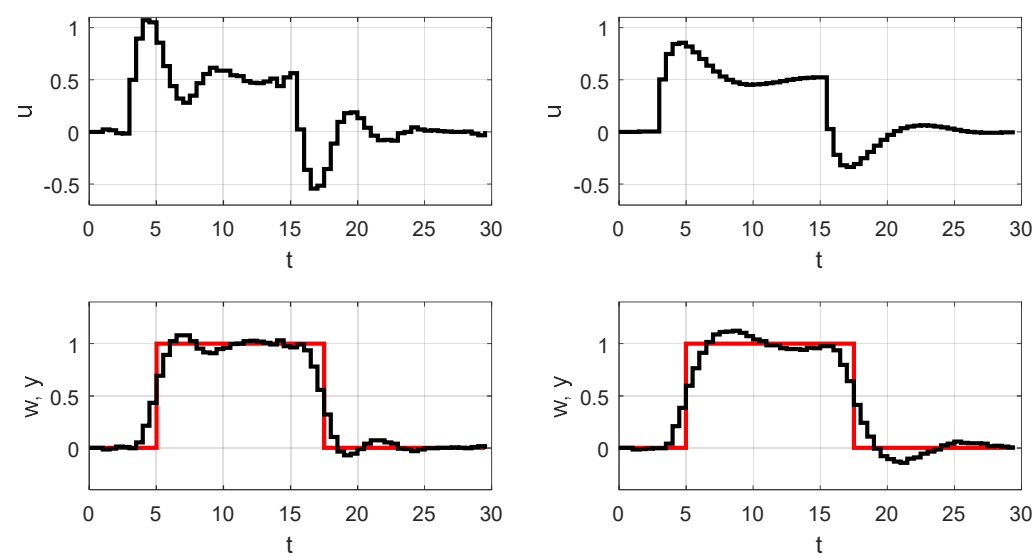

Fig. 4. Simulated control responses without (left) and with filtering polynomial (right) 
Two versions according to the filtering polynomial are applied - without filtering polynomial $(C=1)$ and with second order filtering polynomial $\mathrm{C}=\left(1-0.8 \mathrm{z}^{-1}\right)^{2}$. Simulated control responses are in Fig. 4 and real control responses in Fig. 5.
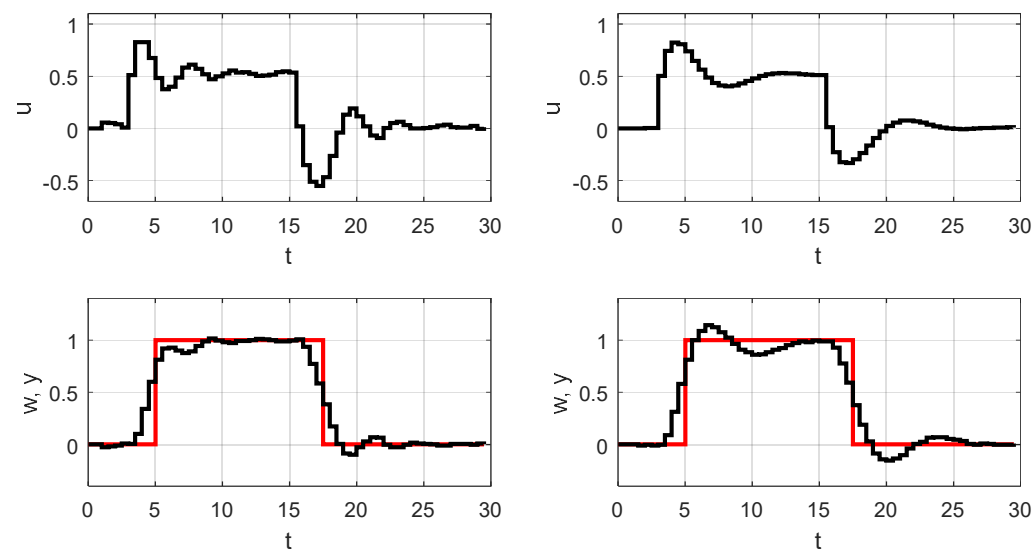

Fig. 5. Real control responses without (left) and with filtering polynomial (right)

\section{Conclusion}

Two fast and easy to use symbolic forms of MPC are presented and applied in laboratory scale. Controllers are parametrized with first order process model parameters $a_{1}$ and $b_{1}$, one penalty parameter $q$ and two filtering polynomial parameters $c_{1}$ and $c_{2}$. Penalty parameter allows to tune the control quality. Filtering parameters can be seen also as tunable parameters. The controller is quite sensitive to measurement noise without filtering $\left(c_{1}=c_{2}=0\right)$. First order polynomial (e.g. $\left.c_{1}=-0.8, c_{2}=0\right)$ will give smoother control actions. Second order polynomial (e.g. $c_{1}=-1.6, c_{2}=0.64$ ) filter improves control actions even more but also increases risk of oscillations. Some trade-off in filtering tuning is necessary similarly to state observing problem.

Acknowledgment: This research was supported by SGS project Modern technology for large-volume data processing and optimal control of technological processes (in Czech) at FEI, University of Pardubice. This support is very gratefully acknowledged.

\section{References}

1. Camacho, E.F., Bordons, C.: Model predictive control. Springer, New York (2004).

2. Rossiter, J.A.: Model-based predictive control: a practical approach. CRC Press, Boca Raton (2003).

3. Soroush, M., Muske K.R.: Analytical Model Predictive Control. In: Nonlinear Model Predictive Control. Progress in Systems and Control Theory, vol. 26, Birkhäuser, Basel (2000).

4. Soroush, M.: Plants for which model predictive control admits an analytical solution. In: Proceedings of the American Control Conference, pp. 3745-3750, (2007). 